Check for updates

Cite this: Phys. Chem. Chem. Phys. 2017, 19, 14248

Received 27th February 2017, Accepted 8th May 2017

DOI: $10.1039 / c 7 c p 01288 h$

rsc.li/pccp

\section{Light-induced relaxation dynamics of the ferricyanide ion revisited by ultrafast XUV photoelectron spectroscopy $\dagger$}

\author{
Nicholas Engel, ${ }^{a}$ Sergey I. Bokarev, (D)*b Alexandre Moguilevski, ${ }^{a}$ Azhr A. Raheem, \\ Ruba Al-Obaidi, ${ }^{a}$ Tobias Möhle, (D) ${ }^{b}$ Gilbert Grell, (D) ${ }^{b}$ Katrin R. Siefermann, ${ }^{c}$ \\ Bernd Abel, ${ }^{c}$ Saadullah G. Aziz, ${ }^{d}$ Oliver Kühn, (D) b Mario Borgwardt, ${ }^{\text {a }}$ \\ Igor Yu. Kiyan (D) *a and Emad F. Aziz*aef
}

\begin{abstract}
Photoinduced charge transfer in transition-metal coordination complexes plays a prominent role in photosynthesis and is fundamental for light-harvesting processes in catalytic materials. However, revealing the relaxation pathways of charge separation remains a very challenging task because of the complexity of relaxation channels and ultrashort time scales. Here, we employ ultrafast XUV photoemission spectroscopy to monitor fine mechanistic details of the electron dynamics following optical ligand-to-metal chargetransfer excitation of ferricyanide in aqueous solution. XUV probe light with a time resolution of $100 \mathrm{fs}$, in combination with density functional theory employing the Dyson orbital formalism, enabled us to decipher the primary and subsequently populated electronic states involved in the relaxation, as well as their energetics on sub-picosecond timescales. We find strong evidence for the spin crossover followed by geometrical distortions due to vibronic interactions (Jahn-Teller effect) in the excited electronic states, rather than localization/delocalization dynamics, as suggested previously.
\end{abstract}

\section{Introduction}

Charge transfer reactions in transition-metal coordination complexes induced by light have received much attention during the last few decades. ${ }^{1-4}$ They play an important role throughout different fields of science in solids and soft matter. ${ }^{5}$ For instance, these reactions constitute the basis of light-harvesting mechanisms occurring in materials of natural ${ }^{6-8}$ and synthetic ${ }^{9-12}$ character. Therefore, their deep understanding is of utmost importance for the development of tomorrow's energy sources based on photovoltaics and photocatalysis. ${ }^{13-17}$

\footnotetext{
${ }^{a}$ Joint Laboratory for Ultrafast Dynamics in Solutions and at Interfaces (JULiq), Institute of Methods for Material Development, Helmholtz-Zentrum Berlin, Albert-Einstein-Str. 15, D-12489 Berlin, Germany.

E-mail: igor.kiyan@helmholtz-berlin.de,emad.aziz@helmholtz-berlin.de

${ }^{b}$ Institute of Physics, University of Rostock, Albert-Einstein-Str. 23-24, D-18059 Rostock, Germany. E-mail: sergey.bokarev@uni-rostock.de

${ }^{c}$ Leibniz Institute of Surface Modification and Joint HZB-IOM-Photonics Lab, Permoserstr. 15, D-04318 Leipzig, Germany

${ }^{d}$ Chemistry Department, Faculty of Science, King Abdulaziz University, 21589 Jeddah, Saudi Arabia

${ }^{e}$ Department of Physics, Freie Universität Berlin, Arnimallee 14, 14195 Berlin, Germany

${ }^{f}$ School of Chemistry, Monash University, Clayton 3800, VIC, Australia

$\dagger$ Electronic supplementary information (ESI) available: Pump intensity study, cross correlation measurements, kinetic models, $F$-test of kinetic models, calculated Huang-Rhys factors, calculated IR spectrum. See DOI: 10.1039/c7cp01288h
}

However, charge transfer usually competes with other photoinduced non-radiative loss channels, such as intersystem crossing, internal conversion, and parallel photochemical reactions. Moreover, in solid and soft-matter systems the influence of the environment may also be decisive for the mechanism of electron transfer, e.g., it may be facilitated by solvent reorganization, as recently discussed in ref. 18 and 19.

In order to derive a general picture of the photophysics of transition metal complexes, it is of importance to understand how the variations of the structure influence the photoinduced dynamics. Ferrocyanide $\left(\left[\mathrm{Fe}(\mathrm{CN})_{6}\right]^{4-}\right)$ and ferricyanide $\left(\left[\mathrm{Fe}(\mathrm{CN})_{6}\right]^{3-}\right)$ ions are perfect simple model systems for this purpose since they resemble the properties of more complex light-harvesting systems such as metal-doped porphyrins. ${ }^{10,13,20-22}$ Although both complexes are very similar in their chemical structure, their photophysics is quite different with respect to the ability to undergo ligand exchange triggered by UV light $(\sim 400 \mathrm{~nm}) .{ }^{23,24}$ For $\left[\mathrm{Fe}(\mathrm{CN})_{6}\right]^{3-}$ in aqueous solution, the quantum yield for exchange of a $\mathrm{CN}^{-}$ligand by $\mathrm{H}_{2} \mathrm{O}$ is 0.016 , which is about an order of magnitude smaller than that for $\left[\mathrm{Fe}(\mathrm{CN})_{6}\right]^{4-} \cdot{ }^{23}$ This indicates a rapid deactivation process for $\left[\mathrm{Fe}(\mathrm{CN})_{6}\right]^{3-}$ which is not accessible for $\left[\mathrm{Fe}(\mathrm{CN})_{6}\right]^{4-}$. While subtle differences in the electronic structure of the two complexes have been discovered in recent years, ${ }^{25-27}$ the origin of the rapid deactivation of photoexcited $\left[\mathrm{Fe}(\mathrm{CN})_{6}\right]^{3-}$ has remained unclear. Unraveling this 
rapid deactivation mechanism calls for an ultrafast experiment, which is sensitive to the electronic structure.

The dynamics following the charge-transfer excitation involving the $\mathrm{CN}^{-}$ligands at a wavelength of $400 \mathrm{~nm}$ was studied by Zhang et al. using UV pump - mid-IR probe spectroscopy. ${ }^{19}$ Assuming that the initial ligand-to-metal charge-transfer (LMCT) transition creates a 'hole' in one ligand, the subsequent dynamics was interpreted in terms of an interplay of charge localization/ delocalization mechanisms within an incoherent interligand hopping model. While the electronic coupling is supposed to determine the rate of electron hopping between equivalent ligands, leading to charge/hole delocalization, geometric relaxation and solvation oppositely lead to charge localization. Based on the observation of single $\mathrm{CN}$-stretch frequencies and anisotropy measurements, Zhang et al. concluded that a fully symmetric excited state was formed as a result of the electron delocalization within the time resolution of $200 \mathrm{fs}$ of their experiment. At later times, signatures of a reduction of molecular symmetry were observed and attributed to charge localization caused by a solvent-assisted structural reorganization of the molecular complex. On even longer timescales in the picosecond range, the excited state was observed to relax back to the electronic ground state.

In the present work, we address the question of relaxation dynamics in ferricyanide by applying transient XUV photoelectron spectroscopy ${ }^{28-30}$ (PES) assisted by density functional theory calculations combined with Dyson orbital formalism. This enabled us to obtain the so far unknown energetics of the excited states involved in LMCT excitation. Furthermore, by probing the electronic structure, the recorded dynamics are directly correlated to the electronic transitions and electronically excited states. The joint analysis of experimental and theoretical data suggests a new interpretation which is consistent with both the IR experimental data obtained by Zhang et al. ${ }^{19}$ and the PES data discussed in this work.

Two fundamental aspects of our work should be emphasized here. First, instead of the qualitative time-domain description introduced in ref. 19, we interpret the observed ultrafast electron dynamics in terms of a well-established picture in the frequency (energy) domain, based on Jahn-Teller theory. ${ }^{31}$ We naturally assume the electronic wave functions to conform to the symmetry of the nuclear frame, thus, implying that the molecular orbitals (MOs) are delocalized on all ligands. The second issue is related to the point group symmetry of $\left[\mathrm{Fe}(\mathrm{CN})_{6}\right]^{3-}$, which was assumed to be fully octahedral $\left(O_{\mathrm{h}}\right)$ in the work by Zhang et al. ${ }^{19}$ and in other previous studies. ${ }^{32-34}$ This assumption is based on the fact that only one band is observed in the IR spectrum, ${ }^{19,34}$ which is assigned to the triply degenerate $t_{1 u} C-N$ stretching vibration mode. However, $\left[\mathrm{Fe}(\mathrm{CN})_{6}\right]^{3-}$ is a $\mathrm{d}^{5}$ low-spin system with uneven populations of the $t_{2 g}$ orbitals and, hence, should experience a weak Jahn-Teller distortion. According to the analysis presented in ref. 35, which is in agreement with the low-temperature magnetic anisotropy measurements, ${ }^{36}$ the vibronic interaction of the $T_{2 g} \otimes\left(e_{g}+t_{2 g}\right)$ type for the doublet ground ${ }^{2} \mathrm{~T}_{2 \mathrm{~g}}$ state with degenerate vibrations leads to a lowering of the symmetry. Two configurations are thus possible having
$D_{4 \mathrm{~h}}$ or $D_{3 \mathrm{~d}}$ symmetry, where the latter geometry yields a larger stabilization energy for $\left[\mathrm{Fe}(\mathrm{CN})_{6}\right]^{3-}$. As we discuss below, only the $D_{3 \mathrm{~d}}$ geometry is consistent with the single IR C-N stretching band. Therefore, this geometry is adopted in our analysis.

The article is organized as follows. After presenting the experimental and theoretical methods, we discuss the experimental data and the fitting procedure employing different kinetic models as well as the results of the calculations. Based on this information, a joint interpretation is given in the Discussion section and summarized in the Conclusions section.

\section{Experimental method}

The experiment was performed with a $500 \mathrm{mM}$ solution of potassium ferricyanide in water. The sample was introduced into the interaction chamber with the use of a liquid micro-jet technique. ${ }^{37}$ The aqueous solution was pushed with the use of a syringe pump through a quartz capillary of $20 \mu \mathrm{m}$ orifice, forming a steady liquid jet with a laminar flow of $\sim 20 \mathrm{~mm}$ length. A small amount ( $10 \mathrm{mM})$ of sodium chloride was admixed with the solution to prevent the streaming potential created due to the friction of liquid flow in the quartz capillary. ${ }^{38}$ Downstream the flow, the volatile sample was caught with a cryogenic trap. This technique enabled to maintaining a low pressure of $1.2 \times 10^{-5} \mathrm{mbar}$ in the experimental chamber, thus, facilitating the detection of photoelectrons ${ }^{28,29}$ and the transmission of XUV light.

The UV-pump and XUV-probe beams were generated by using the $800 \mathrm{~nm}$ (1.55 eV photon energy) output of a Ti:sapphire laser system delivering pulses of $2.5 \mathrm{~mJ}$ energy, 25 fs duration, and $5 \mathrm{kHz}$ repetition rate. The laser output was split into two beams, whereas $60 \%$ of the output power was used to generate the XUV probe pulses via frequency up-conversion of the $800 \mathrm{~nm}$ fundamental beam in a high-order harmonic generation (HHG) process, induced in a cell pressurized with $\sim 30$ mbar of argon. Our HHG setup has been described in detail elsewhere. ${ }^{39}$ For the present experiment, the twenty-first harmonic of $32.55 \mathrm{eV}$ photon energy was selected as the probe using a zone-plate monochromator, yielding a typical XUV pulse duration of $50 \mathrm{fs}$ after monochromatization. The selected beam was focused into the experimental chamber and onto the sample with the use of a toroidal mirror. The focal spot size of $60 \mu \mathrm{m}$ was measured using a razor blade and the photon flux was attenuated to $\sim 10^{6}$ photons per pulse to avoid detector saturation. The remaining $40 \%$ of the laser power was used for generating the pump pulses. For the present experiment, the second harmonic generation with a central wavelength of $400 \mathrm{~nm}$ was utilized. The pump pulses were loosely focused onto a spot size of $\sim 100 \mu \mathrm{m}$, and the peak fluence was set to approximately $18 \mathrm{~mJ} \mathrm{~cm} \mathrm{~cm}^{-2}$ to minimize multiphoton excitation (see ESI, $\dagger$ Fig. S1). The time delay between the pump and probe pulses was controlled with a precision of 1 fs by means of an optical delay stage installed in the pump beam path.

The pump and probe beams were spatially and temporally overlapped on the micro-jet positioned in front of the skimmer 
of a commercial time-of-flight (TOF) electron spectrometer (SPECS, model THEMIS $1000^{40}$ ). The small skimmer size of $0.4 \mathrm{~mm}$ enabled maintaining a low gas pressure of $10^{-6} \mathrm{mbar}$ inside the spectrometer during the operation. The photoelectrons ejected from the jet travel $0.5 \mathrm{~mm}$ through the experimental chamber until they enter the skimmer. Under these conditions, electron scattering off the residual gas molecules is negligible for the detected electrons. The spectrometer was operated in two modes. In the drift mode, corresponding to a conventional fieldfree design, the acceptance angle of the spectrometer is $\pm 1^{\circ}$, the energy resolution is $25 \mathrm{meV}$ at electron kinetic energy of $20 \mathrm{eV}$, and the detectable kinetic energy range is not limited. This mode was used to acquire photoelectron spectra in a wide kinetic energy range, where ionization contributions of both solvent and solute are present. In the wide-angle collection mode, the acceptance angle is increased to $\pm 15^{\circ}$ by using an intrinsic electrostatic lens. This leads to a significant increase of signal, whereas the energy resolution is reduced to $0.1 \mathrm{eV}$. In this configuration, the kinetic energy scale is limited to a certain range around the chosen pass energy. The wide-angle mode was used to acquire spectra with a high signal-to-noise ratio in the energy range that contains the transient signal. The spectrometer is equipped with a low-energy filter, consisting of a metal grid mounted in front of the detector. By applying a negative potential to the grid, the large amount of slow electrons is not admitted to the detector, thus, preventing from detector saturation.

The electron flight time is converted to electron kinetic energy and subsequently to electron binding energy. For photoionization from liquid-phase media, the binding energy $E_{\mathrm{b}}$ is calculated by subtracting the kinetic energy $E_{\text {kin }}$ from the XUV photon energy $E_{\mathrm{ph}}$, analogously to photoionization from gasphase media. Fig. 1 shows the steady-state XUV spectrum of ferricyanide aqueous solution, recorded in the drift operational mode of the spectrometer while applying only the probe beam. The electron distribution is presented on the scale of binding energies. Emission contributions from different orbitals of water and from $\left[\mathrm{Fe}(\mathrm{CN})_{6}\right]^{3-}$ and the counterion $\mathrm{K}^{+}$are indicated

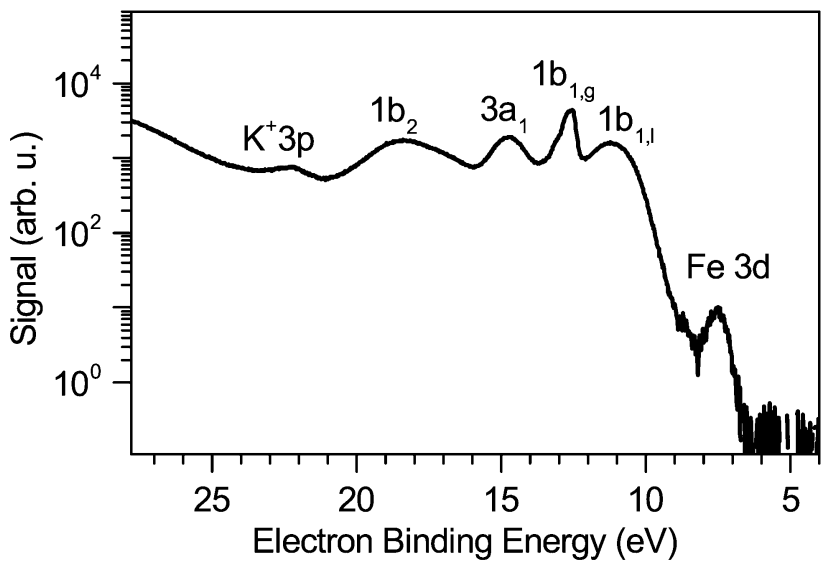

Fig. 1 XUV photoemission spectrum of ferricyanide aqueous solution Spectral peaks are attributed to ionization from the iron-center-localized Fe $3 d$ orbitals, $K^{+} 3 p$, and the $1 b_{1}, 3 a_{1}$, and $1 b_{2}$ water orbitals. in the figure. One can see that the yield from the Fe $3 \mathrm{~d}$ orbital of ferricyanide is pronounced as a well-resolved energy peak in the spectrum. Its central position at $7.4 \mathrm{eV}$ binding energy is in agreement with the previously reported value. ${ }^{41}$

The time response of the experimental setup was inferred from a cross-correlation experiment on the liquid sample. When the pump and the probe pulses overlap in time, multiphoton ionization occurs that involves absorption of an XUV probe photon and simultaneous absorption or emission of one or more pump photons. This process has a non-resonant character and is analogous to laser-assisted photoemission from surfaces previously described in the literature. ${ }^{42,43}$ The ionization yield integrated over a sideband of the laser-assisted ionization process represents a cross-correlation signal of the pump and probe pulses. By recording the photoelectron yield in the range of higher kinetic energies as a function of time delay, we obtained a cross-correlation trace with a width of $103 \mathrm{fs}$ (FWHM) in the first sideband, representing a convolution of the UV-pump and XUV-probe pulse durations (see the ESI $\dagger$ for further details). The peak position of the cross-correlation trace defines time zero.

\section{Theoretical approach}

\section{Electronic structure}

All calculations were performed at the density functional theory (DFT) level employing the range-separated LC-BLYP functional, ${ }^{44}$ where the range separation parameter was optimized according to the $\triangle \mathrm{SCF}$ procedure described in detail in ref. 45 and 46 . For $\left[\mathrm{Fe}(\mathrm{CN})_{6}\right]^{3-}$, the optimal value of this parameter was found to be $0.27 \mathrm{Bohr}^{-1}$. The use of optimally-tuned range-separation functional allows achieving a higher reliability for the absorption and especially photoelectron spectra due to the mitigation of the electron self-interaction error. ${ }^{47}$ All DFT calculations have been performed using the GAUSSIAN $09^{48}$ package employing the aug-cc-pVTZ basis set. ${ }^{49-51}$ This relatively large basis set with an additional set of diffuse functions was applied to reproduce the diffuse wave function of this highly negatively charged ion. The solvent environment was accounted for by using the polarizable continuum model ${ }^{52}$ which was also essential for stabilizing the molecular complex which autoionizes without solvation. The geometry for the doublet $(S=1 / 2)$ ground $\mathrm{D}_{0}$ state (low-spin $\mathrm{d}^{5}$ system) has been optimized resulting in $D_{3 \mathrm{~d}}$ point group symmetry, whereas the quartet state $(S=3 / 2)$ equilibrium geometry has $D_{4 \mathrm{~h}}$ symmetry. A number of doublet and quartet excited states have been calculated at the level of time-dependent DFT (TDDFT).

\section{Photoelectron spectra}

To interpret the experimental data, photoelectron probe spectra have been computed for the ground and lowest LMCT and ligand-field (LF) doublet as well as LF quartet states as these levels are possibly populated after the absorption of the pump pulse prior to photoionization. For most of the calculations, the initial states populated in the Franck-Condon region 
( $D_{0}$ equilibrium geometry) were considered. For the lowest quartet $\mathrm{Q}_{1}$ state, the photoelectron spectrum has also been computed for the respective equilibrium geometry of $D_{4 \mathrm{~h}}$ symmetry. The singlet $(S=0)$, triplet $(S=1)$, and quintet $(S=2)$ electronic states (101 states in each manifold) of the residual $\left[\mathrm{Fe}(\mathrm{CN})_{6}\right]^{2-}$ ion, created in the process of XUV ionization have been considered as final states. The respective electronic structure calculations have been performed using the Tamm-Dancoff approximation to TDDFT employing the aug-cc-pVTZ basis set and PCM as described above. The photoionization cross sections were calculated employing the Dyson orbital (DO) approach as described in ref. 53. The numerical evaluation of the boundcontinuum transition matrix elements has been performed using the ezDyson v3.0 program. ${ }^{54}$ The final state of the photoelectron is therein represented by a plane wave expanded in terms of spherical waves up to angular momentum truncated at $l_{\max }=7$. The numerical integration was carried out on a three-dimensional uniform grid in a box with a side length of $12 \AA$ and 360 grid points per dimension. Since the ionization intensities are proportional to the respective squared DO norms, they were calculated only if the respective values were larger than $10^{-4}$.

\section{Results}

\section{Time-resolved studies}

The transient signal (TS) was derived by subtracting the XUV negative-delay spectra, averaged over the range of applied negative time delays (probe pulse arrives first to the interaction region), as a background from the pump-probe spectra. The two-dimensional plot in Fig. 2 shows the dependency of TS on the binding energy and the time delay. The negative value of the TS in the energy range between 7 and $8 \mathrm{eV}$ is due to the depletion of the Fe $3 \mathrm{~d}$ orbital induced by the pump beam. At short time delays within $\pm 50 \mathrm{fs}$, a strong contribution from the cross-correlation signal is present in the data set. This contribution

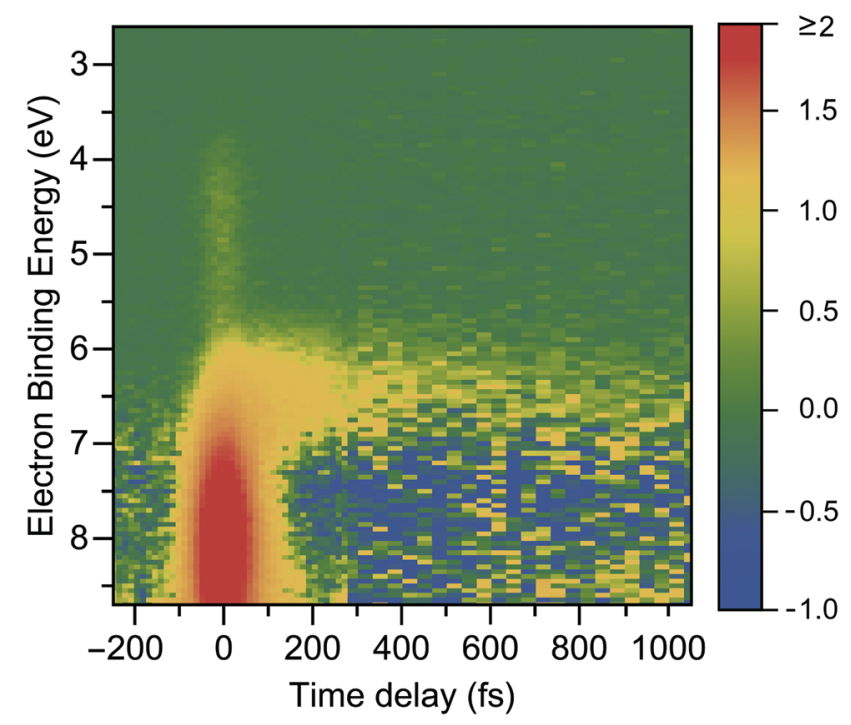

Fig. 2 Transient photoemission signal as a function of the electron energy and the time delay. is due to the first- and second-order cross-correlation sidebands which arise at energies above and below $6.5 \mathrm{eV}$, respectively, as described in the ESI. $\dagger$ The feature of primary interest in Fig. 2 is the TS that appears immediately after time zero in the energy range between 6 and $6.8 \mathrm{eV}$ and disappears on a picosecond time scale. This signal is due to the population and the subsequent relaxation of the LMCT state. It is interesting to note that the central energy of the excited LMCT band is rather close to the ionization energy of $6.11 \mathrm{eV}$ of ferrocyanide, $\left[\mathrm{Fe}(\mathrm{CN})_{6}\right]^{4-}$, defined by emission from the iron-donated $\mathrm{Fe}\left(\mathrm{t}_{2 \mathrm{~g}}\right)$ orbital. $^{41}$ One can expect this, since the iron atom has the same oxidation state in the LMCT excited state of ferricyanide and in the ground state of ferrocyanide molecules.

The time evolution of the TS was found to be independent of energy in the range that encompasses the LMCT band. This was derived by comparing the integrated ionization yield for a number of energy intervals of $0.2 \mathrm{eV}$ width lying between 6 and $6.8 \mathrm{eV}$ binding energies. Therefore, in the analysis presented below, we consider the yield integrated over the entire LMCT band. Its time dependence is presented in Fig. 3. One can immediately see that the signal at time zero has an asymmetric shape, indicating the presence of a short-lived excited state. However, the consideration of the population and decay of a single excited state is inadequate to reproduce the data presented in Fig. 3. This is inferred from the $F$-test of different kinetic models and supported by the theoretical predictions.

In the simple model, we consider the electron population dynamics of a single excited state $\mathrm{X}_{1}$ :

$$
\mathrm{GS} \stackrel{P(t)}{\longrightarrow} \mathrm{X}_{1} \stackrel{k_{1}}{\longrightarrow} \mathrm{R},
$$

where GS denotes the initial ground state, $P(t)$ is the excitation rate proportional to the intensity of the pump pulse, and $k_{1}$ is

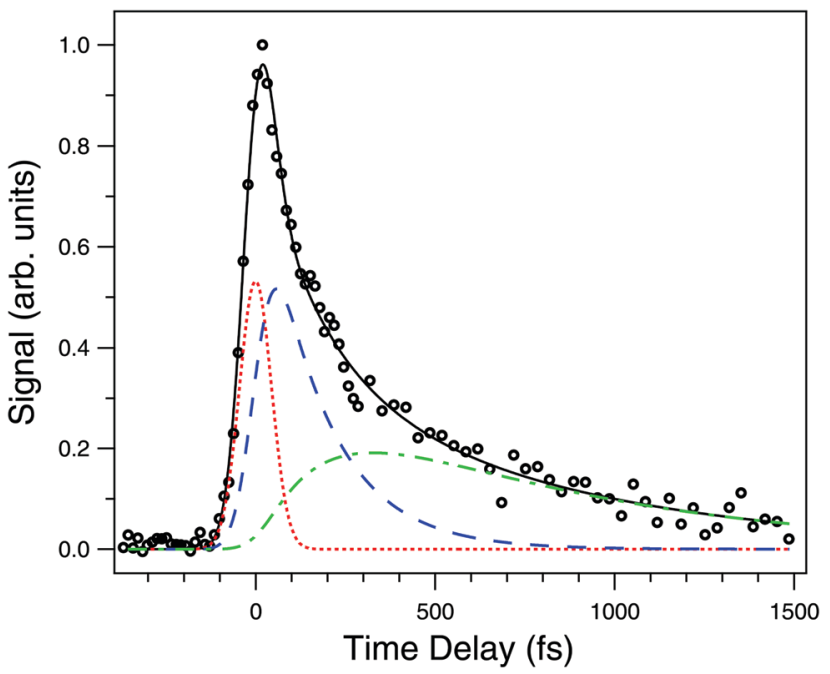

Fig. 3 Transient signal integrated over the energy range between 6 and $6.8 \mathrm{eV}$ encompassing the LMCT band. The black solid line represents a fit of the extended model (eqn (2)) to the experimental data (shown by circles). Using the fit, the overall signal is decomposed to the individual ionization yields of states $X_{1}$ and $X_{2}$ and the cross-correlation signal, shown by the dashed (blue), dot-dashed (green), and dotted (red) lines, respectively. 
the decay rate to a relaxed state $\mathrm{R}$. The latter is not specified and it is considered that the XUV ionization yield of $\mathrm{R}$ does not contribute to the TS at energies of interest between 6 and $6.8 \mathrm{eV}$. In the extended model, an additional excited state $\mathrm{X}_{2}$ is involved in the sequential relaxation pathway:

$$
\mathrm{GS} \stackrel{P(t)}{\longrightarrow} \mathrm{X}_{1} \stackrel{k_{1}}{\longrightarrow} \mathrm{X}_{2} \stackrel{k_{2}}{\longrightarrow} \mathrm{R} .
$$

The systems of differential equations and their analytical solutions, describing the transient population of the excited state(s) in reactions (1) and (2), respectively, are presented in the ESI. $\dagger$ To describe the time dependency of the XUV ionization yield of the excited state(s), the transient population was convoluted with the temporal profile of the probe pulse, and the convolution result was fitted to the experimental data shown in Fig. 3. The cross-correlation signal was taken into account in the fit procedure as well.

By comparing the fit results obtained for the simple and the extended kinetic models, we find that the $F$ statistic significantly exceeds a critical value defined by the $F$-distribution function (see ESI $\dagger$ ). This implies that the simple model can be rejected with high confidence and the scheme (2) needs to be considered instead. Based on the fit results, the decomposition of the integrated TS to the individual ionization yields of states $\mathrm{X}_{1}$ and $\mathrm{X}_{2}$ and the crosscorrelation signal is shown in Fig. 3. The decay time constants of $\mathrm{X}_{1}$ and $\mathrm{X}_{2}$, representing the values of ${k_{1}}^{-1}$ and $k_{2}{ }^{-1}$, are $170 \mathrm{fs}$ and $730 \mathrm{fs}$, respectively.

\section{DFT-calculations}

The equilibrium structure of the molecule in the doublet ground electronic state is slightly distorted from the octahedral geometry and has $D_{3 \mathrm{~d}}$ point group symmetry. Six of the NC-Fe-CN valence angles are $88.3^{\circ}$ and the other six are $91.7^{\circ}$. The ground electronic state has symmetry $\mathrm{A}_{1 \mathrm{~g}}$ and the electronic configuration $\left(\mathrm{d}_{x^{2}-y^{2}}\right)^{2}\left(\mathrm{~d}_{x y}\right)^{2}\left(\mathrm{~d}_{z^{2}}\right)^{1}\left(\mathrm{~d}_{x z}\right)^{0}\left(\mathrm{~d}_{y z}\right)^{0}$. There are two more closely lying electronic states of $\mathrm{E}_{\mathrm{g}}$ symmetry, where either of the degenerate $\mathrm{e}_{\mathrm{g}}$ orbitals $\left(\mathrm{d}_{x^{2}-y^{2}}\right.$ or $\left.\mathrm{d}_{x y}\right)$ are singly occupied. These two states are $0.22 \mathrm{eV}$ above the ground state and, thus, at room temperature only the ground state is appreciably populated. The calculated ground state IR spectrum is presented in the ESI. $\dagger$ It involves a number of slightly different $\mathrm{C}-\mathrm{N}$ stretching frequencies which, however, form a single absorption band due to the spectral line broadening. Thus, the $D_{3 \mathrm{~d}}$ symmetry is consistent with the observations reported in ref. 19 and 34 .

Symmetry selection rules imply that dipole transitions from the ground $\mathrm{A}_{1 \mathrm{~g}}$ state should be to states with ungerade symmetry. This means that local ligand-field $d \rightarrow d$ transitions are forbidden and only LMCT or MLCT states should be "bright" and seen in the absorption spectrum. The form of the spectrum below $5 \mathrm{eV}$ is determined by few LMCT transitions of the $\sigma \pi_{\mathrm{CN}} \rightarrow \mathrm{d}_{z^{2}}$ type, see Fig. 4 .

According to the $F$-test analysis, there is a need to include one more state, $\mathrm{X}_{2}$, in the interpretation of the results of transient experiments. As is apparent from Fig. 4 showing both the experimental and computed electronic absorption spectra of $\left[\mathrm{Fe}(\mathrm{CN})_{6}\right]^{3-}$, the excitation can populate three bright states

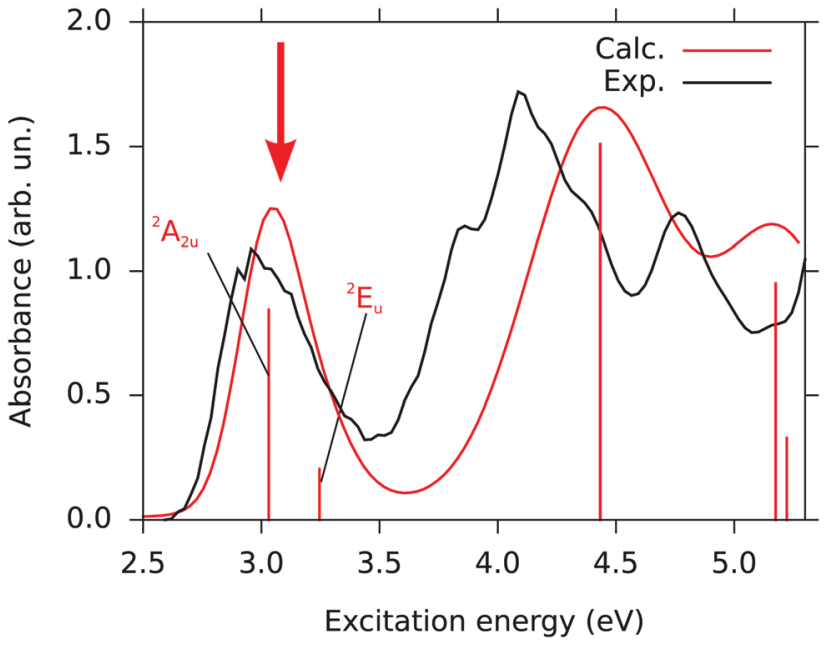

Fig. 4 Comparison of the experimental and calculated absorption spectra of $\left[\mathrm{Fe}(\mathrm{CN})_{6}\right]^{3-}$ in water. The experimental spectrum is taken from ref. 26. The photon energy of the pump pulse is denoted with a red arrow. Transitions to the three bright states ${ }^{2} \mathrm{~A}_{2 \mathrm{u}}$ and doubly-degenerate ${ }^{2} \mathrm{E}_{\mathrm{u}}$ are possible.

forming a band at 2.6-3.5 eV with asymmetric lineshape. Note that the probability to populate the ${ }^{2} \mathrm{~A}_{2 \mathrm{u}}$ state is 0.67 and the fraction of both ${ }^{2} \mathrm{E}_{\mathrm{u}}$ states according to the calculated oscillator strengths is 0.33 . In addition, the population from ${ }^{2} \mathrm{E}_{\mathrm{u}}$ should quickly decay to ${ }^{2} \mathrm{~A}_{2 \mathrm{u}}$, since both states are notably non-adiabatically coupled via the pseudo-Jahn-Teller mechanism involving $\mathrm{Fe}-\mathrm{CN}$ stretching $\left(374 \mathrm{~cm}^{-1}\right)$ and $\mathrm{NC}-\mathrm{Fe}-\mathrm{CN}$ deformation $\left(447 \mathrm{~cm}^{-1}\right)$ vibrational modes of $\mathrm{e}_{\mathrm{g}}$ symmetry (see ESI $\dagger$ ). Although the ${ }^{2} \mathrm{E}_{\mathrm{u}} \rightarrow{ }^{2} \mathrm{~A}_{2 \mathrm{u}}$ relaxation could interfere with the processes discussed below, it is expected to occur within the cross-correlation time what makes this channel rather difficult to disentangle experimentally. Accordingly, in our interpretation, we assume that the excited state dynamics start from the ${ }^{2} \mathrm{~A}_{2 \mathrm{u}}$ state.

The analysis of the vibrational modes on the basis of the shifted harmonic oscillator model ${ }^{5}$ shows a number of tuning modes with non-zero Huang-Rhys factors (see Table S1 in the ESI $\dagger$ ). The most interesting mode is the $\mathrm{a}_{1 \mathrm{~g}} \mathrm{Fe}-\mathrm{CN}$ stretching one with a ground state frequency of $384 \mathrm{~cm}^{-1}$, because it is active for transitions between states of the doublet manifold as well as between doublet and quartet states. The respective 1D cut through the potential energy surfaces is shown in Fig. 5(a). Other tuning modes yield qualitatively similar cuts and are disregarded in the following interpretation for simplicity.

Remarkably, very close to the Franck-Condon region, there is an almost barrierless intersection between the potential energy surfaces of the ${ }^{2} \mathrm{~A}_{2 \mathrm{u}}$ and the lowest-lying quartet ${ }^{4} \mathrm{~B}_{1 \mathrm{~g}}$ state, cf. Fig. 5(a). The potential energy surfaces of LMCT states also cross those of LF doublet states, with the crossing point, however, lying higher than the excitation energy denoted in Fig. 5(a) with a dashed horizontal line. In Fig. 5(b), the calculated photoelectron spectra are presented for different electronic states of $\left[\mathrm{Fe}(\mathrm{CN})_{6}\right]^{3-}$. Note that no explicit water molecules were included in the theoretical calculations and thus the background is not present in this case. The ground state spectrum essentially represents a peak from the occupied iron 3d-orbitals at approximately 

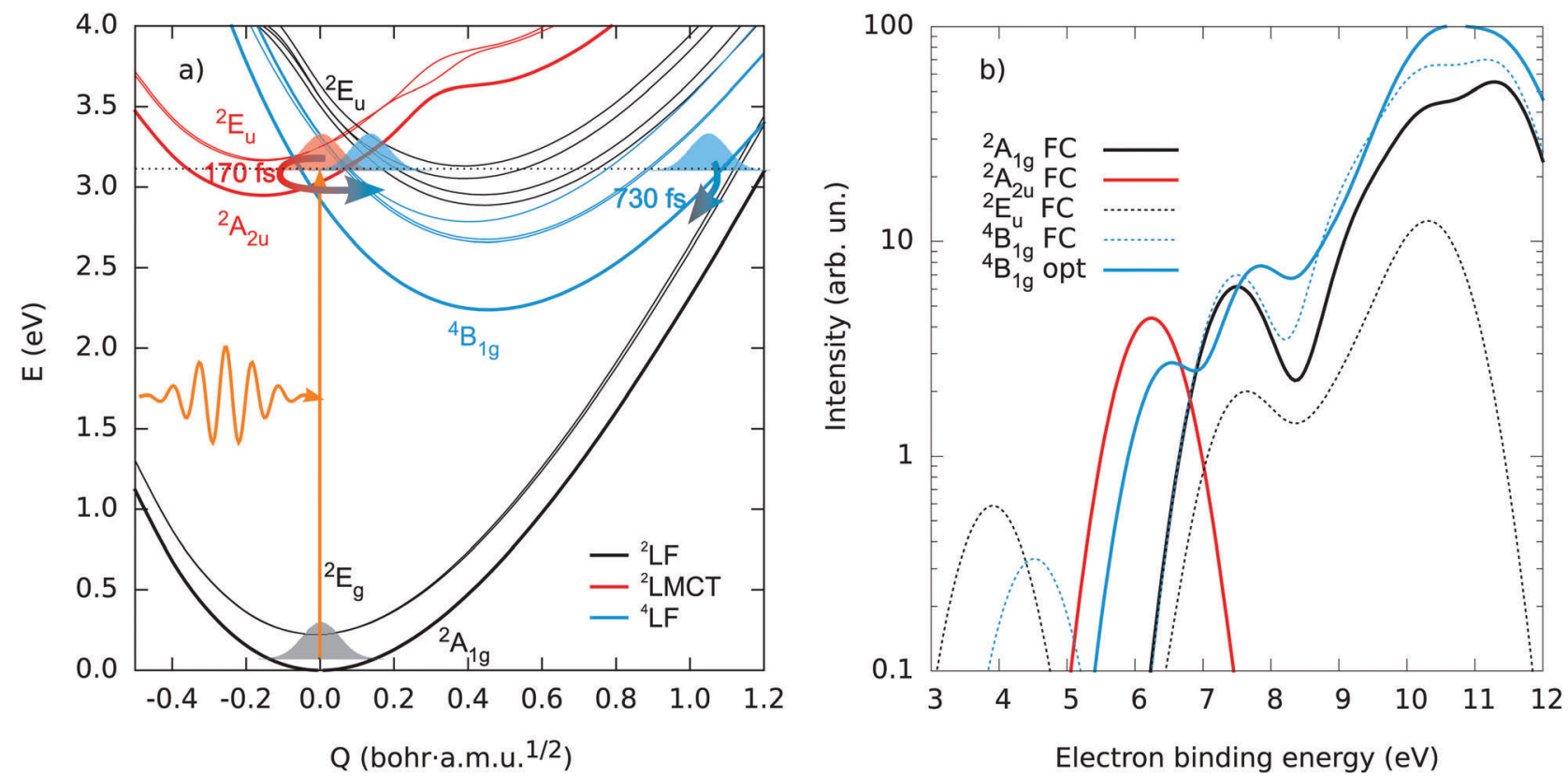

Fig. 5 (a) Calculated $1 \mathrm{D}$ cuts through the potential energy surfaces of the lowest doublet and quartet electronic states of $\left[\mathrm{Fe}(\mathrm{CN})_{6}\right]^{3-}$ along the $\mathrm{a}_{1 \mathrm{~g}} \mathrm{Fe}-\mathrm{CN}$ stretching mode with a ground state frequency of $384 \mathrm{~cm}^{-1}$. Black lines correspond to the doublet ligand-field states ( $\left.{ }^{2} L F\right)$, red lines represent doublet charge-transfer states ( ${ }^{2} \mathrm{LMCT}$ ) populated by the pump pulse (orange arrow) with energy marked by the horizontal dashed line, and cyan lines represent quartet ligand-field states ( ${ }^{4} \mathrm{LF}$ ). The three main states involved in eqn (2) are denoted with thicker lines. (b) Calculated PES originating from different electronic states. Color code coincides with panel (a). FC denotes the Franck-Condon point corresponding to the orange arrow in panel (a) and opt denotes the optimized geometry of the ${ }^{4} B_{1 g}$ lowest quartet state.

$7.4 \mathrm{eV}$ and a broad band consisting of transitions from a combination of iron and ligand orbitals above $8.5 \mathrm{eV}$. For the interpretation of the experimental data, the most interesting energy region is below $6.4 \mathrm{eV}$, where the transient signals from the excited states are observed, as discussed in the following section.

\section{Discussion}

We employ transient XUV photoelectron spectroscopy for the first time here with 100 fs time resolution to monitor the mechanistic details of the electron dynamics following the optical LMCT excitation of ferricyanide in a liquid water micro-jet. Ferricyanide is a well-suited model system for more complex coordination systems in which similar relaxation channels are present. In the experiment, two very fast and hitherto unresolved sub-picosecond timescales were extracted, suggesting the presence of one "dark" state on the way from the initially prepared LMCT state back to the ground state. Notably, the transient signals correspond to a single binding energy. This is an important experimental observable, only accessible using the present technique and very valuable for deciphering the relaxation pathway.

The joint analysis of the experimental and theoretical data strongly suggests the following interpretation of the ultrafast photophysical processes in $\left[\mathrm{Fe}(\mathrm{CN})_{6}\right]^{3-}$, initiated by $3.1 \mathrm{eV}$ photoexcitation. The initially excited ${ }^{2} \mathrm{~A}_{2 \mathrm{u}}$ state gives rise to the transient signal in the 6-7 eV energy range (Fig. 5(b)). (Here, an unresolved rapid ${ }^{2} \mathrm{E}_{\mathrm{u}} \rightarrow{ }^{2} \mathrm{~A}_{2 \mathrm{u}}$ process is assumed.) The Franck-Condon region is close to a crossing point between the potential curves of the ${ }^{2} \mathrm{~A}_{2 \mathrm{u}}$ and ${ }^{4} \mathrm{~B}_{1 \mathrm{~g}}$ states. The ${ }^{2} \mathrm{~A}_{2 \mathrm{u}}$ potential in the Franck-Condon region has a relatively small gradient and the initially excited wave packet moves only slowly away from this point. This yields favorable conditions for an ultrafast spin crossover between these two states, which takes place within approximately $170 \mathrm{fs}$. An alternative explanation would involve the motion of the wave packet on the ${ }^{2} \mathrm{~A}_{2 \mathrm{u}}$ surface with population transfer occurring every time it returns to the Franck-Condon region. At the harmonic frequency of $384 \mathrm{~cm}^{-1}$, the period would be $\sim 90 \mathrm{fs}$ which is approximately a factor of two smaller than the fitted time constant of $170 \mathrm{fs}$. However, on the basis of the signal-tonoise ratio of the measured data we cannot provide evidence for such a cumulative population transfer.

The gradient in the newly populated ${ }^{4} \mathrm{~B}_{1 g}$ state is large, causing a rapid elongation of the $\mathrm{Fe}-\mathrm{CN}$ bonds and, thus, the wave packet quickly leaves the crossing point. The PES of the ${ }^{4} \mathrm{~B}_{1 g}$ state at the Franck-Condon point is essentially different from that of the ${ }^{2} \mathrm{~A}_{2 u}$ state. A spectral band should appear at notably lower binding energies of 4-5 eV. However, upon further geometrical reorganization the emission spectrum significantly changes, with the band position shifting back to the $6-7 \mathrm{eV}$ range. Thus, because of the minute population time of the Franck-Condon quartet configuration, this signal was not detected in the noisy background. Instead, the signal from the geometrically distorted quartet state is detected, which approximately coincides with the original short-living signal from ${ }^{2} \mathrm{~A}_{2 \mathrm{u}}$ in energy (see solid red and cyan lines in Fig. 5(b)). Due to the relatively small size of the molecule, the vibrational cooling occurs on longer time 
scales than those considered here ${ }^{55-57}$ and the excess of vibrational energy (see the dashed horizontal line in Fig. 5(a)) in the ${ }^{4} \mathrm{~B}_{1 \mathrm{~g}}$ state should be sufficient to reach the crossing region with the ${ }^{2} \mathrm{E}_{\mathrm{g}}$ states which further relax to the ${ }^{2} \mathrm{~A}_{1 \mathrm{~g}}$ ground state. The respective transition represents the deactivation channel assigned to the second measured time constant of $730 \mathrm{fs}$. The calculated PES for the doublet ligand field ${ }^{2} \mathrm{E}_{\mathrm{u}}$ state (dashed black curve in Fig. 5(b)) excludes its involvement in the dynamics since it has notably different spectral features. As mentioned above, the time evolution of the TS is independent of energy in the range of signal appearance. Therefore, we believe that the $730 \mathrm{fs}$ timescale should not be assigned to the solvent-mediated localization of the hole at one CN ligand as suggested in ref. 19. Such localization should be possible only upon removal of the substantial excess of vibrational energy, which should in turn lead to the binding energy shifts in the PES spectrum. However, further investigations are needed to verify this point.

Our findings are in agreement with those of Zhang et al., ${ }^{19}$ suggesting the reduction of symmetry and occurrence of new bands. However, an alternative interpretation arises here. The appearance of the new transient IR absorption bands after $200 \mathrm{fs}$ in their experiments may simply be explained by the Jahn-Teller distortion we found in the quartet ${ }^{4} B_{1 g}$ state exhibiting a minimum with $D_{4 \mathrm{~h}}$ point group symmetry. In this case, axial and equatorial $\mathrm{Fe}-\mathrm{CN}$ bonds have different lengths, resulting in two distinct $\mathrm{C}-\mathrm{N}$ stretching IR absorption bands. Further changes are caused by the transition to the ${ }^{2} \mathrm{E}_{\mathrm{g}}$ low-lying ligand-field states and to the electronic ground state with $D_{4 \mathrm{~h}}$ symmetry, which is a metastable state of higher energy than that of $D_{3 \mathrm{~d}}$ symmetry. The observed symmetry breaking can be explained solely by the vibronic interactions in the excited electronic states without involving a spatial localization/delocalization model proposed in ref. 19.

\section{Conclusions}

In the present contribution, we have highlighted the power of femtosecond extreme UV photoelectron spectroscopy to measure the early electron dynamics following the optical excitation of ferricyanide in a liquid microjet, i.e., in aqueous solution. From the analysis of our data involving time-resolved traces and elaborated quantum-chemical calculations, we conclude that both nonadiabatic and spin crossover transitions occur on a sub-picosecond timescale. Due to a direct probe of the absolute electron energies (relative to the vacuum level) and high time-resolution, as well as different selection rules (in comparison to photon detection methods), the current approach provides unique and very important complementary information in comparison to other techniques, such as transient absorption and emission studies.

Interestingly, our findings and conclusions are in agreement with the data of Zhang et al., ${ }^{19}$ however, we strongly suggest an alternative interpretation. Our theoretical approach and overall analysis revealed that the geometrical distortions due to vibronic interactions (Jahn-Teller effect) in the excited electronic states are responsible for the observed transients in the previous studies, rather than localization/delocalization dynamics originally suggested by Zhang et al. ${ }^{19}$

In summary, the joint analysis of experimental and theoretical data represent a powerful tool for ultrafast electron transfer reactions in the condensed phase that are otherwise difficult to measure, and this analysis suggests a novel interpretation of the seemingly well-known ultrafast photophysics of the ferricyanide transition-metal complex in aqueous solution. Most importantly, we show here that the initial doublet-quartet intersystem crossing takes place at $170 \mathrm{fs}$ and is followed by slower (730 fs) intersystem crossing to the lower excited and ground states.

In conclusion, the new tool highlighted here gives access to new observables significantly different and complementary to optical spectroscopy, also see ref. 30, and allows us to literally shed new light on light-harvesting systems more complex than ferricyanide, no matter whether they are natural or synthetic.

\section{Acknowledgements}

This work was funded by the European Research Council, Grant No. 279344, by the Helmholtz-Gemeinschaft via the VH-NG-635 Grant, and by the Deanship of Scientific Research (DSR), King Abdulaziz University, Jeddah, Grant No. D-003-435.

\section{References}

1 A. Vogler and H. Kunkely, Coord. Chem. Rev., 2000, 208, 321-329.

2 H. Xu, R. Chen, Q. Sun, W. Lai, Q. Su, W. Huang and X. Liu, Chem. Soc. Rev., 2014, 43, 3259.

3 N. A. Smith and P. J. Sadler, Philos. Trans. R. Soc., A, 2013, 371, 20120519.

$4 \mathrm{H}$. Yersin, Transition Metal and Rare Earth Compounds, Springer Berlin Heidelberg, 2004.

5 V. May and O. Kühn, Charge and Energy Transfer Dynamics in Molecular Systems, Weinheim: Wiley-VCH, 2011.

6 J. N. Gehlen, M. Marchi and D. Chandler, Science, 1994, 263, 499-502.

7 S. R. Meech, A. J. Hoff and D. A. Wiersma, Proc. Natl. Acad. Sci. U. S. A., 1986, 83, 9464-9468.

8 E. Collini, C. Y. Wong, K. E. Wilk, P. M. G. Curmi, P. Brumer and G. D. Scholes, Nature, 2010, 463, 644-647.

9 S. Nishitani, N. Kurata, Y. Sakata, S. Misumi, A. Karen, T. Okada and N. Mataga, J. Am. Chem. Soc., 1983, 105, 7771-7772.

10 M. R. Wasielewski, Chem. Rev., 1992, 92, 435-461.

11 H. Kurreck and M. Huber, Angew. Chem., Int. Ed., 1995, 34, 849-866.

12 E. Collini and G. D. Scholes, Science, 2009, 323, 369-373.

13 D. Gust, T. A. Moore and A. L. Moore, Acc. Chem. Res., 2009, 42, 1890-1898.

14 C. Deibel, T. Strobel and V. Dyakonov, Adv. Mater., 2010, 22, 4097-4111. 
15 A. Marchioro, J. Teuscher, D. Friedrich, M. Kunst, R. van de Krol, T. Moehl, M. Grätzel and J.-E. Moser, Nat. Photonics, 2014, 8, 250-255.

16 G. F. Moore and G. W. Brudvig, Annu. Rev. Condens. Matter Phys., 2011, 2, 303-327.

17 J. Yu, J. Jin, B. Cheng and M. Jaroniec, J. Mater. Chem. A, 2014, 2, 3407.

18 A. D. Miller, I. Bezel, K. J. Gaffney, S. Garrett-Roe, S. H. Liu, P. Szymanski and C. B. Harris, Science, 2002, 297, 1163-1166.

19 W. Zhang, M. Ji, Z. Sun and K. J. Gaffney, J. Am. Chem. Soc., 2012, 134, 2581-2588.

20 M. R. Wasielewski, J. Org. Chem., 2006, 71, 5051-5066.

21 A. Kay and M. Graetzel, J. Phys. Chem., 1993, 97, 6272-6277.

22 H. Imahori, Y. Mori and Y. Matano, J. Photochem. Photobiol., $C$, 2003, 4, 51-83.

23 M. Fuller, K. Lebrocq, E. Leslie and I. Wilson, Aust. J. Chem., 1986, 39, 1411-1419.

24 M. Shirom and G. Stein, J. Chem. Phys., 1971, 55, 3379-3382.

25 T. Lee, Y. Jiang, C. G. Rose-Petruck and F. Benesch, J. Chem. Phys., 2005, 122, 084506.

26 M. Reinhard, T. J. Penfold, F. A. Lima, J. Rittmann, M. H. Rittmann-Frank, R. Abela, I. Tavernelli, U. Rothlisberger, C. J. Milne and M. Chergui, Struct. Dyn., 2014, 1, 024901.

27 K. Kunnus, W. Zhang, M. G. Delcey, R. V. Pinjari, P. S. Miedema, S. Schreck, W. Quevedo, H. Schröder, A. Föhlisch, K. J. Gaffney, M. Lundberg, M. Odelius and P. Wernet, J. Phys. Chem. B, 2016, 120, 7182-7194.

28 O. Link, E. Lugovoy, K. Siefermann, Y. Liu, M. Faubel and B. Abel, Appl. Phys. A: Mater. Sci. Process., 2009, 96, 117-135.

29 M. Faubel, K. R. Siefermann, Y. Liu and B. Abel, Acc. Chem. Res., 2012, 45, 120-130.

30 A. Moguilevski, M. Wilke, G. Grell, S. I. Bokarev, S. G. Aziz, N. Engel, A. A. Raheem, O. Kühn, I. Y. Kiyan and E. F. Aziz, ChemPhysChem, 2017, 18, 465-469.

31 I. Bersuker, The Jahn-Teller Effect, Cambridge University Press, Cambridge, 2010.

32 J. J. Alexander and H. B. Gray, J. Am. Chem. Soc., 1968, 90, 4260-4271.

33 K. Pierloot, E. Van Praet, L. G. Vanquickenborne and B. O. Roos, J. Phys. Chem., 1993, 97, 12220-12228.

34 G. Prampolini, P. Yu, S. Pizzanelli, I. Cacelli, F. Yang, J. Zhao and J. Wang, J. Phys. Chem. B, 2014, 118, 14899-14912.

35 M. Atanasov, P. Comba, C. A. Daul and A. Hauser, J. Phys. Chem. A, 2007, 111, 9145-9163.

36 J. Baker and B. Figgis, Aust. J. Chem., 1982, 35, 265-275.

37 A. Charvat, E. Lugovoj, M. Faubel and B. Abel, Rev. Sci. Instrum., 2004, 75, 1209-1218.

38 N. Kurahashi, S. Karashima, Y. Tang, T. Horio, B. Abulimiti, Y.-I. Suzuki, Y. Ogi, M. Oura and T. Suzuki, J. Chem. Phys., 2014, 140, 174506.

39 J. Metje, M. Borgwardt, A. Moguilevski, A. Kothe, N. Engel, M. Wilke, R. Al-Obaidi, D. Tolksdorf, A. Firsov, M. Brzhezinskaya,
A. Erko, I. Y. Kiyan and E. F. Aziz, Opt. Express, 2014, 22, 10747.

40 See the website http://www.specs.de/.

41 R. Seidel, S. Thürmer, J. Moens, P. Geerlings, J. Blumberger and B. Winter, J. Phys. Chem. B, 2011, 115, 11671-11677.

42 L. Miaja-Avila, C. Lei, M. Aeschlimann, J. L. Gland, M. M. Murnane, H. C. Kapteyn and G. Saathoff, Phys. Rev. Lett., 2006, 97, 113604.

43 G. Saathoff, L. Miaja-Avila, M. Aeschlimann, M. M. Murnane and H. C. Kapteyn, Phys. Rev. A: At., Mol., Opt. Phys., 2008, 77, 022903.

44 H. Iikura, T. Tsuneda, T. Yanai and K. Hirao, J. Chem. Phys., 2001, 115, 3540-3544.

45 O. S. Bokareva, G. Grell, S. I. Bokarev and O. Kühn, J. Chem. Theory Comput., 2015, 11, 1700-1709.

46 S. I. Bokarev, O. S. Bokareva and O. Kühn, Coord. Chem. Rev., 2015, 304-305, 133-145.

47 R. Baer, E. Livshits and U. Salzner, Annu. Rev. Phys. Chem., 2010, 61, 85-109.

48 M. J. Frisch, G. W. Trucks, H. B. Schlegel, G. E. Scuseria, M. A. Robb, J. R. Cheeseman, G. Scalmani, V. Barone, B. Mennucci, G. A. Petersson, H. Nakatsuji, M. Caricato, X. Li, H. P. Hratchian, A. F. Izmaylov, J. Bloino, G. Zheng, J. L. Sonnenberg, M. Hada, M. Ehara, K. Toyota, R. Fukuda, J. Hasegawa, M. Ishida, T. Nakajima, Y. Honda, O. Kitao, H. Nakai, T. Vreven, J. A. Montgomery, Jr., J. E. Peralta, F. Ogliaro, M. Bearpark, J. J. Heyd, E. Brothers, K. N. Kudin, V. N. Staroverov, R. Kobayashi, J. Normand, K. Raghavachari, A. Rendell, J. C. Burant, S. S. Iyengar, J. Tomasi, M. Cossi, N. Rega, J. M. Millam, M. Klene, J. E. Knox, J. B. Cross, V. Bakken, C. Adamo, J. Jaramillo, R. Gomperts, R. E. Stratmann, O. Yazyev, A. J. Austin, R. Cammi, C. Pomelli, J. W. Ochterski, R. L. Martin, K. Morokuma, V. G. Zakrzewski, G. A. Voth, P. Salvador, J. J. Dannenberg, S. Dapprich, A. D. Daniels, O. Farkas, J. B. Foresman, J. V. Ortiz, J. Cioslowski and D. J. Fox, Gaussian09 Revision D.01, Gaussian Inc., Wallingford, CT, 2009.

49 T. H. Dunning, J. Chem. Phys., 1989, 90, 1007-1023.

50 N. B. Balabanov and K. A. Peterson, J. Chem. Phys., 2005, 123, 064107.

51 R. A. Kendall, T. H. Dunning Jr. and R. J. Harrison, J. Chem. Phys., 1992, 96, 6796-6806.

52 J. Tomasi, B. Mennucci and R. Cammi, Chem. Rev., 2005, 105, 2999-3093.

53 G. Grell, S. I. Bokarev, B. Winter, R. Seidel, E. F. Aziz, S. G. Aziz and O. Kühn, J. Chem. Phys., 2015, 143, 074104.

54 S. Gozem and A. I. Krylov, http://iopenshell.usc.edu, ezDyson, 2015.

55 J. Aßmann, M. Kling and B. Abel, Angew. Chem., Int. Ed., 2003, 42, 2226-2246.

56 R. von Benten, A. Charvat, O. Link, B. Abel and D. Schwarzer, Chem. Phys. Lett., 2004, 386, 325-329.

57 R. S. von Benten and B. Abel, Chem. Phys., 2010, 378, 19-26. 